\title{
Bilateral Encrusted Metallic Stent Successfully Removed by Ureteroscopic Lithotripsy Using a Ho:YAG Laser in a Patient with Malignant Myeloma
}

\author{
Takahiro Hanai Takashi Kawahara Hiroaki Ishida Shinnosuke Kuroda \\ Toshitaka Miyai Masato Yasui Shuntaro Aoki Hiroji Uemura \\ Departments of Urology and Renal Transplantation, Yokohama City University Medical \\ Center, Yokohama, Japan
}

\section{Keywords}

Bilateral ureteral stent encrustation - Metallic ureteral stent · Resonance · Encrustation .

Ureteroscopy

\begin{abstract}
Ureteral stent encrustation is sometimes encountered, especially in cases in which a ureteral stent has been forgotten. An 84-year-old female patient with malignant myeloma underwent metallic ureteral stent insertion to treat malignant ureteral obstruction. At the time of scheduled ureteral stent exchange, the stent was heavily encrusted and could not be removed on either side. We performed endoscopic lithotripsy to remove the encrusted ureteral stents. The bilaterally encrusted metallic ureteral stents were successfully removed using Ho:YAG laser lithotripsy after inserting another ureteral stent placement besides the encrusted metallic ureteral stents.

\section{Introduction}

Ureteral stents were first reported by Zimskind et al. [1] in 1967. Nowadays, ureteral stents are fundamental urological devices that are used to preserve renal function from severe hydronephrosis due to ureteral obstruction.

We previously reported the usefulness of ureteral stenting in the treatment of malignant ureteral obstruction (MUO) and that hematological disease was responsible for $2.6 \%$ of all cases of MUO [2, 3]. In malignant myeloma, steroid treatment is sometimes introduced with 

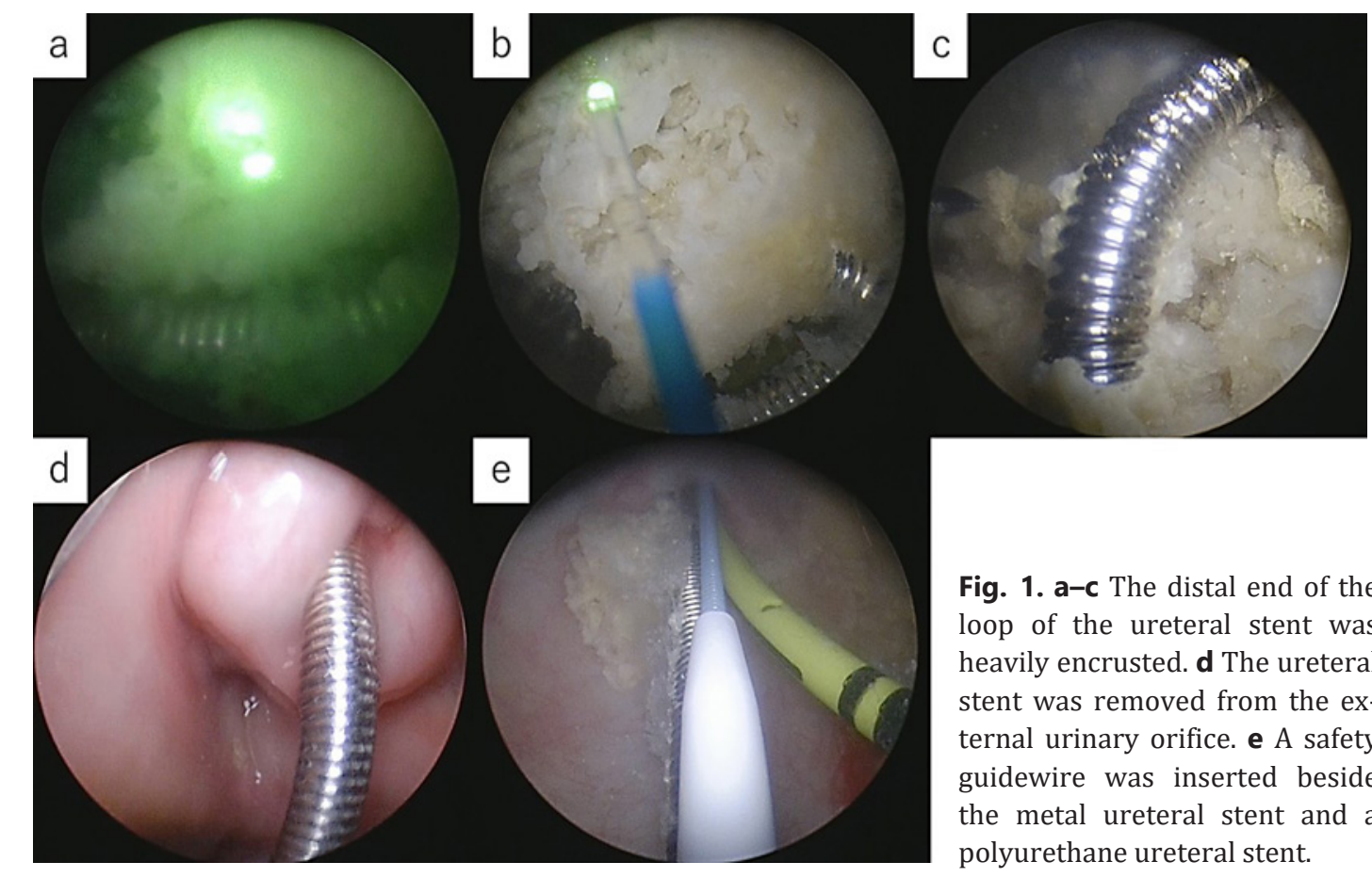

Hanai et al.: Bilateral Metallic Ureteral Stent Encrustation

Fig. 1. a-c The distal end of the loop of the ureteral stent was heavily encrusted. $\mathbf{d}$ The ureteral stent was removed from the external urinary orifice. e A safety guidewire was inserted beside the metal ureteral stent and a polyurethane ureteral stent.

systemic chemotherapy. Steroid use is a risk factor for ureteral stone development [4]. Thus, the management of encrusted ureteral stents is important, especially for patients with longterm indwelling stents. Metallic ureteral stents have been reported to be useful in terms of long-term tolerance and effectiveness [5].

We herein report a case in which an encrusted metallic ureteral stent was successfully removed by Ho:YAG laser lithotripsy after bilateral ureteral stent placement.

\section{Case Presentation}

An 84-year-old woman with malignant myeloma was referred to our department due to decreasing renal function. CT revealed slight bilateral hydronephrosis, and the patient's renal function increased after bilateral insertion of metallic ureteral stents. At the time of scheduled metallic ureteral stent exchange, the ureteral stents were heavily encrusted bilaterally; thus, we could not exchange the ureteral stents and decided to insert ureteral stents bilaterally beside the encrusted metallic ureteral stents on both sides.

We first observed and tried to grasp the encrusted metallic stent to remove it from the external ureteral orifice; however, it could not be removed. Due to the strong encrustation on the distal end of the metallic stent, we first performed lithotripsy. Then, we advanced the distal end of the metallic stent though to the external ureteral orifice (Fig. 1a-d). We inserted a guidewire beside the metallic ureteral stent as a safety sheath (Fig. 1e). We inserted another guidewire using a polyurethane stent and inserted a 10-/12-Fr ureteral access sheath, using this guidewire, at the L4 level, and observed the encrusted ureteral stent using flexible ureteroscopy. We performed lithotripsy using a Ho:YAG laser to remove the encrusted stones, taking care to avoid damaging the metallic ureteral stent. During lithotripsy, we continuously applied tension to remove the metallic stent. After lithotripsy, the metallic ureteral stent was successfully removed (Fig. 2). While observing the upper ureter and renal pelvis, we performed lithotripsy for large stones. Finally, we inserted a polyurethane ureteral stent.

\section{Karger'}




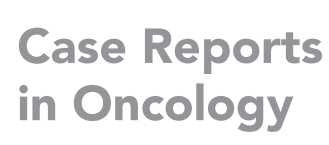

\begin{tabular}{l|l}
\hline Case Rep Oncol 2020;13:1501-1505 \\
\hline DOI: 10.1159/000511601 & $\begin{array}{l}\text { ○ 2020 The Author(s). Published by S. Karger AG, Basel } \\
\text { www.karger.com/cro }\end{array}$ \\
\hline
\end{tabular}

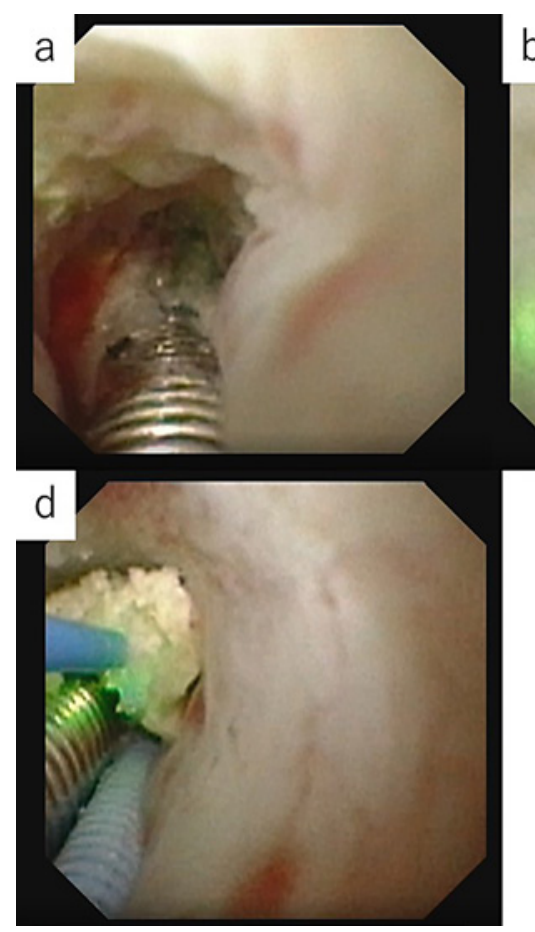

Hanai et al.: Bilateral Metallic Ureteral Stent Encrustation

Fig. 2. a-d Surface stones of the encrusted ureteral stent were removed by lithotripsy using a Ho:YAG laser.
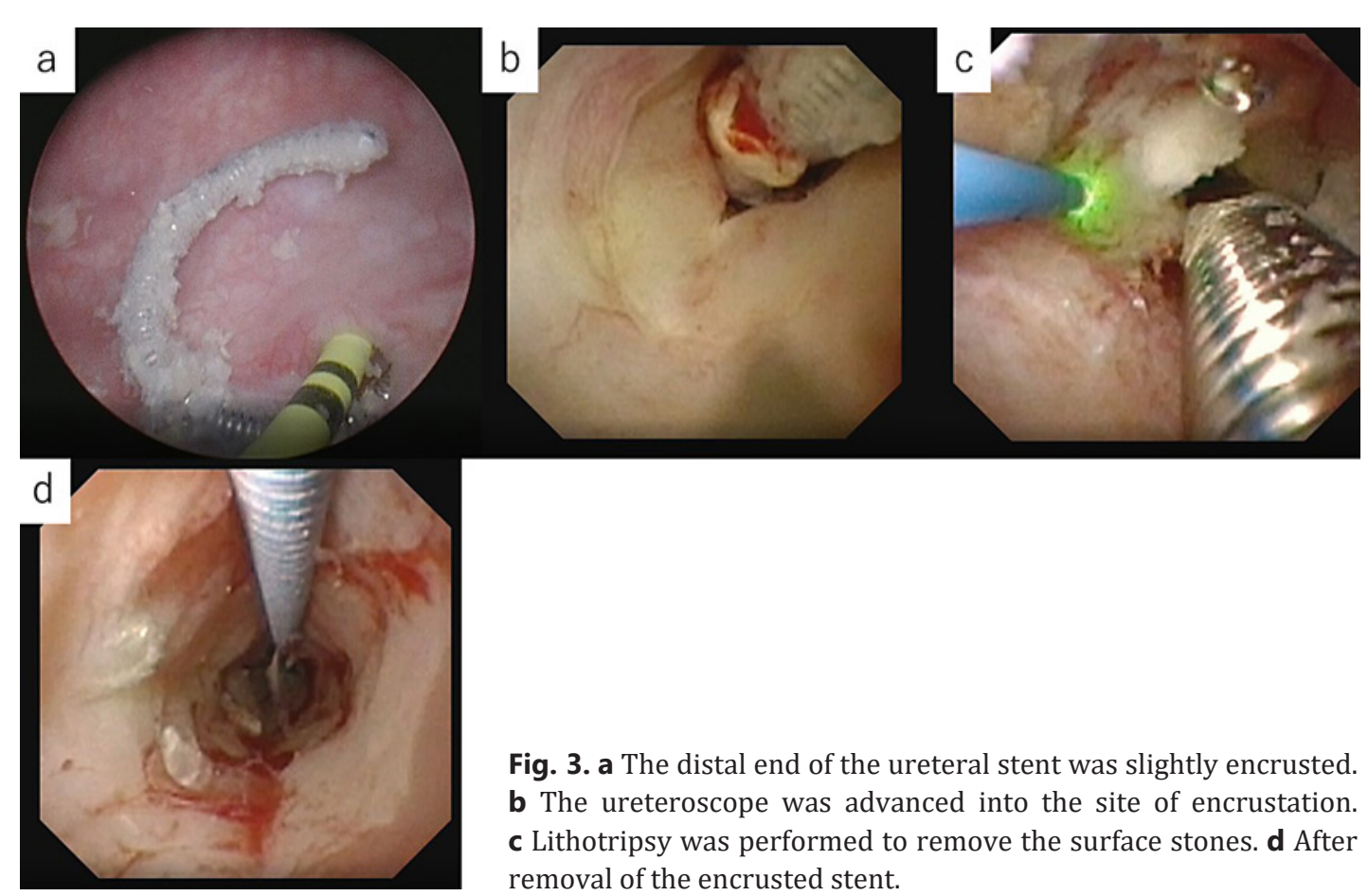

Fig. 3. a The distal end of the ureteral stent was slightly encrusted. b The ureteroscope was advanced into the site of encrustation. c Lithotripsy was performed to remove the surface stones. d After removal of the encrusted stent.

We then performed the same procedure on the left side. On the left side, the encrustation was not so severe. We removed the distal end of the stent from the external ureteral orifice. We then inserted a safety guidewire and inserted a ureteral access sheath beside the safety guidewire. We performed lithotripsy before the successful removal of the encrusted metallic ureteral stent (Fig. 3). As we had done on the other side, we concluded the procedure by inserting a polyurethane ureteral stent.

\section{Karger'}


To reduce the risk of encrustation, scheduled ureteral stent exchange will be performed every 2 months.

\section{Discussion}

In cases of MUO, ureteral stenting allows percutaneous nephrostomy, an invasive procedure, to be avoided. Metallic ureteral stent insertion was associated with a superior and more durable response in comparison to polyurethane ureteral stent insertion [6,7]. Thus, metallic ureteral stent insertion can increase the time without nephrostomy in patients with MUO. There are some reports of cases of polyurethane stent-resistant MUO in which metallic ureteral stent insertion allowed percutaneous nephrostomy to be avoided [8].

We previously reported the correlation between ureteral stent indwelling time and the incidence of ureteral stent encrustation [4]. The detailed mechanism of ureteral stent encrustation has not been established. However, a possible mechanism is that biofilm forms around the ureteral stent and that stones develop from this biofilm [4, 9]. Multiple stent materials and stent designs have been developed; however, ureteral stent encrustation can occur with any type of ureteral stent [5]. No studies have reported differences in encrustation between polyurethane and metallic ureteral stents. At our institute, a total of 118 metallic ureteral stents have been inserted. The present patient represents the second case of heavy encrustation and the first case of bilateral encrustation [10]. In our experience, metallic ureteral stents are easily damaged by Ho:YAG lasers due to the unique spiral form. Thus, lithotripsy should be carefully performed.

We performed Ho:YAG laser lithotripsy to remove an encrusted metallic stent. In the present case, a ureteral stent was inserted beside the encrusted ureteral stent. The addition of this ureteral stent facilitated dilatation of the ureter and thus contributed to inserting a ureteral access sheath, which provided more working space for flexible ureteroscopy and facilitated drainage of the fragmented tiny stones from around the metallic stent. Application of this procedure via the percutaneous approach for the removal of heavily encrusted ureteral stents has sometimes been reported. However, the patient in the present case required heparinization due to pulmonary embolism. Thus, an endourological approach was initially chosen.

The insertion of a ureteral stent beside the encrusted ureteral stent and performance of laser lithotripsy may be useful when we encounter the situation of a ureteral stent that is difficult to remove via a transurethral approach.

\section{Conclusion}

Bilaterally encrusted metallic ureteral stents were successfully removed with Ho:YAG laser lithotripsy after the bilateral insertion of ureteral stents.

\section{Statement of Ethics}

The patient provided written informed consent for the publication of this study including images, and ethical approval was obtained.

\section{Karger'}




\section{Conflict of Interest Statement}

The authors declare no conflicts of interest in association with the present study.

\section{Funding Sources}

No funding was received.

\section{Author Contributions}

T.H. and T.K. drafted the manuscript. All authors performed the procedure.

\section{References}

1 Zimskind PD, Fetter TR, Wilkerson JL. Clinical use of long-term indwelling silicone rubber ureteral splints inserted cystoscopically. J Urol. 1967;97(5):840-4.

2 Ohtaka M, Kawahara T, Takamoto D, Mochizuki T, Hattori Y, Teranishi JI, et al. Gastrointestinal cancer and bilateral hydronephrosis resulted in a high risk of ureteral stent failure. BMC Urol. 2018;18(1):35.

3 Kawahara T, Takamoto D, Miyoshi Y, Yao M, Uemura H. Inserting a metallic ureteral stent using an antegrade approach freed a patient from nephrostomy created due to hydronephrosis caused by bladder cancer. Case Rep Oncol. 2019;12(2):560-3.

4 Kawahara T, Ito H, Terao H, Yoshida M, Matsuzaki J. Ureteral stent encrustation, incrustation, and coloring: morbidity related to indwelling times. J Endourol. 2012;26(2):178-82.

5 Kawahara T. Editorial Comment to Ureteric stents: overview of current clinical applications and economic implications. Int J Urol. 2020;27(1):16.

6 Kawahara T. Editorial Comment from Dr Kawahara to Prevention and treatment of symptoms associated with indwelling ureteral stents: a systematic review. Int J Urol. 2017;24(4):260.

7 Elsamra SE, Leavitt DA, Motato HA, Friedlander JI, Siev M, Keheila M, et al. Stenting for malignant ureteral obstruction: tandem, metal or metal-mesh stents. Int J Urol. 2015;22(7):629-36.

8 Mochizuki T, Kawahara T, Iwamoto G, Kuroda S, Yao M, Uemura H. The use of a metal stent in the treatment of ureteral stricture in a patient who had undergone radical cystectomy with the creation of an ileal conduit. Case Rep Oncol. 2018;11(1):159-63.

9 Kawahara T, Miyamoto H, Ito H, Terao H, Uemura H, Kubota Y, et al. Discolored ureteral stents: findings in urinalysis and urine culture. PLoS One. 2015;10(4):e0122984.

10 Kawahara T, Kobayashi K, Kurora S, Yao M, Uemura H. Successful removal of an encrusted metallic ureteral stent using a disposable ureteroscope and Ho:YAG laser lithotripsy. Urolithiasis. 2020;48(3):281-2. 